\title{
Methylphenidate Modifies the Motion of the Circadian Clock
}

\author{
Michael C Antle*, , 2,3, Hester C van Diepen ${ }^{3}$, Tom Deboer ${ }^{3}$, Pardis Pedram ${ }^{3}$, Rob Rodrigues Pereira ${ }^{4}$ and \\ Johanna H Meijer ${ }^{2}$ \\ 'Department of Psychology, Hotchkiss Brain Institute, University of Calgary, Calgary, AB, Canada; ${ }^{2}$ Department of Physiology and Pharmacology, \\ Hotchkiss Brain Institute, University of Calgary, Calgary, AB, Canada; ${ }^{3}$ Department of Molecular Cell Biology, Leiden University Medical Centre, \\ Leiden, The Netherlands; ${ }^{4}$ Department of Pediatrics, Maastad Hospital, Rotterdam, The Netherlands
}

\begin{abstract}
People with attention-deficit/hyperactivity disorder (ADHD) often experience sleep problems, and these are frequently exacerbated by the methylphenidate they take to manage their ADHD symptoms. Many of the changes to sleep are consistent with a change in the underlying circadian clock. The present study was designed to determine if methylphenidate alone could alter properties of the circadian clock. Young male mice were examined in light-dark cycles and in constant darkness and recordings were performed on behavioral activity, sleep, and electrical activity in the suprachiasmatic nucleus (SCN) of freely moving mice. Methylphenidate in the drinking water $(0.08 \%)$ significantly increased activity in the mid-to-late night, and led to a delay in the onset of activity and sleep relative to the light-dark cycle. While locomotor levels returned to baseline after treatment ended, the phase angle of entrainment required at least a week to return to baseline levels. In constant darkness, the free-running period of both wheel-running and general locomotor rhythms was lengthened by methylphenidate. When the treatment ended, the free-running period either remained stable or only partially reverted to baseline levels. Methylphenidate also altered the electrical firing rate rhythms in the SCN. It induced a delay in the trough of the rhythm, an increment in rhythm amplitude, and a reduction in rhythm variability. These observations suggest that methylphenidate alters the underlying circadian clock. The observed changes are consistent with clock alterations that would promote sleep-onset insomnia. Neuropsychopharmacology (2012) 37, 2446-2455; doi:I0.1038/npp.20I2.103; published online 4 July 2012
\end{abstract}

Keywords: ritalin; SCN; sleep; in-vivo electrophysiology

\section{INTRODUCTION}

Attention-deficit/hyperactivity disorder (ADHD) is characterized by inattention, hyperactivity, and impulsiveness, symptoms that closely resemble those observed following sleep deprivation (Pilcher and Huffcutt, 1996; Sadeh, 2007; Van der Heijden et al, 2005). Children (Owens et al, 2009) and adults (Boonstra et al, 2007; Sobanski et al, 2008; Van Veen et al, 2010) with ADHD frequently exhibit sleep problems, including shorter sleep duration, difficulty waking up, daytime sleepiness, increased nocturnal movements, longer sleep latencies, and more nocturnal awakening. These symptoms affect a large proportion of adults with ADHD, with $65 \%$ complaining of always having trouble sleeping and $87 \%$ reporting difficulty going to bed on time (Boonstra et al, 2007). When examining the subset of ADHD patients with sleep-onset insomnia, additional symptoms are observed, most notably delayed onset and offset of sleep, delayed dim-light melatonin onset, and an attenuated amplitude of the rest-activity cycle (Novakova et al, 2011;

*Correspondence: Dr MC Antle, Department of Psychology, University of Calgary, 2500 University Drive NW, Calgary, AB, Canada T2N IN4, Tel: + | 40324 | 5819, Fax: + | 4032828249 ,

E-mail: antlem@ucalgary.ca

Received I March 2012; revised 9 May 2012; accepted 30 May 2012
Van Veen et al, 2010), all symptoms consistent with a disruption of the circadian clock. Supporting this is the recent observation that a polymorphism associated with the gene clock, one of the molecular 'gears' of the intracellular circadian clock, may be a contributing factor for adult ADHD (Kissling et al, 2008).

Methylphenidate is an amphetamine derivative and is the first choice medical treatment for ADHD in both adults and children. It acts as a reuptake inhibitor at both dopamine and norepinephrine transporters, increasing levels of these neurotransmitters in the synapse (Kodo et al, 2010). While methylphenidate is quite effective at managing the inattention, impulsiveness, and hyperactivity associated with ADHD, it may not help the co-morbid sleep problems, and in fact methylphenidate may exacerbate these symptoms. In children, placebo-controlled studies have demonstrated that methylphenidate leads to longer sleep latency, lower sleep efficiency, decreased total sleep time (Corkum et al, 2008; Galland et al, 2010; Sangal et al, 2006; Schwartz et al, 2004), increased motor activity at sleep onset, decreased circadian amplitude, and phase delayed daily rhythms (Ironside et al, 2010). Many of these symptoms are consistent with either a phase delayed circadian clock or a circadian clock with a lengthened period. It has long been appreciated that amphetamines, such as methamphetamine, can have a pronounced effect on the rat circadian clock, 
lengthening period, and disrupting entrainment to lightdark cycles (Honma et al, 1986). A few studies have demonstrated that methylphenidate can alter the distribution of activity over the day in rats (Algahim et al, 2009; Gaytan et al, 1997; Lee et al, 2011), although specific parameters such as phase angle of entrainment and freerunning period could not be assessed in these studies, and the circadian clock located in the suprachiasmatic nucleus (SCN; Antle and Silver, 2005) was not examined. The present study was designed to determine if methylphenidate on its own in healthy animals could alter properties of the circadian clock.

\section{MATERIALS AND METHODS}

\section{Animals and Housing}

Male C57BL6 mice (Harlan, Horst, The Netherlands) were either 6 weeks ( $n=45$, behavioral experiment) or 12 weeks ( $n=12$, in-vivo electrophysiology; $n=8$, sleep-wake recordings) of age at the start of the study. Animals in the behavioral part of the study were individually housed in cages equipped with running wheels $(n=24)$, with passive infrared motion sensors (PIRs, $n=12)$, or with both $(n=9)$. Animals in the electrophysiology and sleep part of the study were individually housed in standard mouse cages before recording, and were transferred to large recording chambers (Deboer et al, 2007b; Houben et al, 2009). Animals started the experiment under a $12 \mathrm{~h}: 12 \mathrm{~h}$ lightdark cycle, with ad libitum access to food and drink throughout the experiment. Wheel-running and general locomotion as assessed through PIRs were monitored using a computer running Clocklab data collection software (Actimetrics, Wilmette, IL). Activity counts were summed over each minute. All experiments were approved by the Institutional Animal Studies Committee of the Leiden University Medical Centre.

\section{Drug Preparation}

Methylphenidate (Novartis Pharma B.V., Arnhem, The Netherlands) was dissolved in drinking water at a concentration of $0.8 \mathrm{mg} / \mathrm{ml}$. This dose was based on that found to produce a significant circadian response in SCN lesioned rats (Honma and Honma, 1992). Fresh methylphenidate solution was provided every 3 days at noon.

\section{Behavioral Study 1-Light Dark Cycle}

Mice $(n=12)$ were housed in a 12:12 LD cycle throughout the experiment, and were given normal drinking water for 2 weeks (Baseline). At mid-day on the 15th day, mice were started on either methylphenidate $(0.08 \%, n=6)$ or normal drinking water $(n=6)$. Fresh drinking solution was provided every 3 days at mid-day. This regimen lasted for 3 weeks (Treatment), after which animals were placed back on regular drinking water for a further 2 weeks (Recovery).

Activity records (actograms) were analyzed using the Clocklab Analysis software, with data collected into 10-min bins. Average waveforms of activity profiles were created for each week of the experiment by first averaging the daily activity profile for each animal over 7 days, and then averaging these profiles over each animal in the same treatment group. Quantitative analysis summed the activity in each animal's average waveform into 4-hour blocks. Data from the methylphenidate and water treatment groups were analyzed separately using two-way repeated measures analyses of variance (ANOVAs) with time of day and week of treatment as independent variables. Phase angle of entrainment was analyzed by determining time of activity onset for each day, and averaging the seven onset times for each week. The difference between time of dark onset and activity onset provided the phase angle of entrainment. Phase angle of entrainment data were analyzed using two-way factorial ANOVAs with treatment condition and treatment week as independent variables.

\section{Behavioral Study 2-Constant Darkness}

Mice were housed with either running wheels $(n=12)$ or PIRs $(n=12)$. One mouse in the running wheel condition died before drug treatment began and was excluded from analysis. All mice started with 2 weeks in a 12:12 LD cycle after which they were switched to constant darkness (DD). After 2 weeks of DD (Baseline), half the mice from each housing condition ( $n=6$ wheels, $n=6$ PIRs) were started on methylphenidate $(0.08 \%)$, while the other half from each housing condition ( $n=5$ wheels, $n=6$ PIRs) stayed on regular drinking water. Fresh drinking solution was provided at noon every 3 days. This regimen lasted for 3 weeks (Treatment), after which animals were placed back on regular drinking water for a further 2 weeks (Recovery). Mice were 13 weeks old at the end of the methylphenidate treatment. An additional set of mice $(n=9)$ housed in cages equipped with both running wheels and PIRs were used to examine a dose response to methylphenidate. Baseline, Treatment, and Recovery procedures were identical to those described above, except that mice received $0.02 \%(n=3)$, $0.04 \%(n=3)$, or $0.12 \%(n=3)$ methylphenidate.

Free-running period was calculated for the three treatment blocks (Baseline, Treatment, and Recovery) using a Chi-squared periodogram routine in Clocklab. Given large individual variability in period length in the Baseline block, statistical analysis was performed on period change between blocks (Treatment-Baseline; Recovery-Treatment). Period change data were analyzed using two-way factorial ANOVAs with treatment condition and treatment block as independent variables.

Total activity levels were analyzed by determining the average level of activity per $10 \mathrm{~min}$ bin for each day for each animal. These daily averages were then averaged over all the days in each treatment block (Baseline, Treatment, and Recovery). Activity levels were normalized within each animal relative to their baseline levels. Data from methylphenidate and control animals were analyzed using two-way repeated measures ANOVAs with treatment and treatment block as independent variables.

\section{Sleep-Wake Recordings}

Animals $(n=8)$ were implanted under deep anesthesia (ketamine, $100 \mathrm{mg} / \mathrm{kg}$; xylazine, $20 \mathrm{mg} / \mathrm{kg}$; atropine $1 \mathrm{mg} / \mathrm{kg}$ ) with electroencephalogram (EEG) and electromyogram (EMG) electrodes as described previously (Deboer et al, 
2007b). The electrodes were connected to a miniature receptacle and the assembly was fixed to the skull with two support screws and dental cement. At least 2 weeks were allowed for post surgery recovery.

In a randomized cross-over design, the mice were exposed to methylphenidate in the drinking water $(0.08 \%)$ or regular drinking water for at least 2 weeks starting at about 14 weeks of age. Subsequently, a 24-h baseline day was recorded followed by a 6-h sleep deprivation, starting at lights on, and recovery was recorded for $18 \mathrm{~h}$. Sleep deprivation was carried out by introducing objects (eg, fresh food and nesting material) into the cage, and later by tapping the cage whenever the animals appeared drowsy or the EEG exhibited slow waves (Deboer et al, 2007a; Huber et al, 2000). The mice were never disturbed during feeding and drinking.

To adapt to the recording condition, the mice were connected to the recording system by a flexible cable and a counterbalanced swivel system for 3 days. The EEG and EMG were simultaneously and continuously recorded for $48 \mathrm{~h}$. EEG and EMG were recorded, amplified, filtered, and scored as previously described (Deboer et al, 2007b).

ANOVA served to determine the effect of treatment and sleep deprivation on vigilance states and EEG slow-wave activity (SWA, mean EEG power density between 0.75 and $4.0 \mathrm{~Hz}$ ) during non-rapid eye-movement (NREM) sleep, which is a marker of sleep homeostatic regulation (Achermann and Borbely, 2011). Whenever significant effects were present $(p<0.05)$, paired $t$-tests were used to further evaluate differences.

\section{In-Vivo Electrophysiology}

Animals $(n=12)$ were implanted with tripolar electrodes at about 12 weeks of age. This older age of animal was necessary for the reliability of coordinates and adequate skull thickness. The tripolar electrodes (MS333-3, Plastics One, Roanoke, VA) had two twisted and polyimide-insulated stainless steel electrodes aimed at the SCN, and one uninsulated electrode placed in the cortex as a reference. Animals were anesthetized as described above. Using a stereotaxic frame with digital readout precise to $10 \mu \mathrm{m}$, the skull was leveled both laterally and between bregma and lambda. The electrode was implanted at a $5^{\circ}$ angle, at the same rostrocaudal level as bregma, $0.61 \mathrm{~mm}$ lateral to midline, and $5.38 \mathrm{~mm}$ ventral to dura. The electrode was fixed to the skull using jewellers' screws and dental acrylic. Following surgery, animals were given an analgesic (buprenorphine, $0.1 \mathrm{mg} / \mathrm{kg}$ ) and saline. Animals were allowed 2 weeks to recover before being placed in the recording chamber.

Animals were placed in custom-designed recording chambers and were connected to a recording system with a counterbalanced swivel allowing the animals to freely move during the recording, as described previously (Houben et al, 2009). The electrical activity was amplified and bandwidth filtered $(0.5-5 \mathrm{kHz})$. Window discriminators were used to detect action potentials, which were counted in 10 -s bins. Drinking was monitored by breakage of an infrared beam in front of the sipper tube, while general locomotion was monitored using PIRs. Animals were recorded for 12-15 days, with 4-5 days each of baseline, methylphenidate $(0.08 \%)$, and recovery. Methylphenidate treatment occurred at about 14 weeks of age. At the end of the experiment, brains were collected to confirm electrode placement as described previously (Deboer et al, 2007a).

\section{In-Vivo SCN Electrical Activity Analysis}

In-vivo electrical activity rhythms were smoothed with a penalized least squares algorithm (Eilers, 2003). Trough levels were subtracted from peak levels to determine the amplitude of the electrical activity rhythm. The effect of methylphenidate on amplitude was calculated by dividing mean amplitude levels during methylphenidate treatment by mean amplitude levels during baseline. Phase delays were determined by comparing the trough time during baseline with trough time during methylphenidate treatment. In addition, the variability of the firing rate of the SCN was calculated by averaging the standard deviation of the firing rate during the peak $\pm 3 \mathrm{~h}$ and the trough $\pm 3 \mathrm{~h}$. Variability of the firing rate during baseline was compared with the variability during the first day of methylphenidate treatment. Results were examined with paired $t$-tests. All means are presented in both the text and figures with their SEMs. Threshold for determining significance was set at $p<0.05$.

\section{RESULTS}

\section{Methylphenidate Consumption}

Mice drank $5.08 \pm 0.27 \mathrm{ml}$ of $0.08 \%$ methylphenidate per day. This corresponds to a daily dose of $4.06 \mathrm{mg} /$ day, or $153.3 \mathrm{mg} / \mathrm{kg} /$ day for a $30 \mathrm{-g}$ mouse. Due to spillage, these values are likely an overestimate of the actual amount of drug consumed, and therefore represent the maximal dose.

\section{Behavioral Study 1 - Light Dark Cycle}

Methylphenidate treatment changed the activity profile of the animals, such that activity during the latter half of the night was increased (Figure $1 \mathrm{~b}$ and $\mathrm{d}$ ). The activity levels quickly returned to baseline when methylphenidate treatment was terminated. This increase was significant during the last $8 \mathrm{~h}$ of the night (Treatment $\times$ Time interaction, $F_{(30,150)}=3.01$, $p<0.001$ ). No such change was observed in animals that remained on water throughout (Figure $1 \mathrm{a}$ and $\mathrm{c}$ ).

Control animals maintained a stable phase angle of entrainment throughout the study. By comparison, activity onset was significantly delayed during methylphenidate treatment (main effect of treatment, $F_{(1,50)}=36.865$, $p<0.001$; Figure 2). The phase angle became significantly later over the course of methylphenidate treatment (treatment $\times$ week interaction, $\left.F_{(5,50)}=7.537, p<0.001\right)$. Post hoc analysis revealed that the phase angle of entrainment during the third week of methylphenidate treatment was significantly later than during the first week of methylphenidate treatment.

\section{Behavioral Study 2 - Constant Darkness}

Due to clogged water bottles, two methylphenidate animals were excluded from analysis in the wheel-running 
a

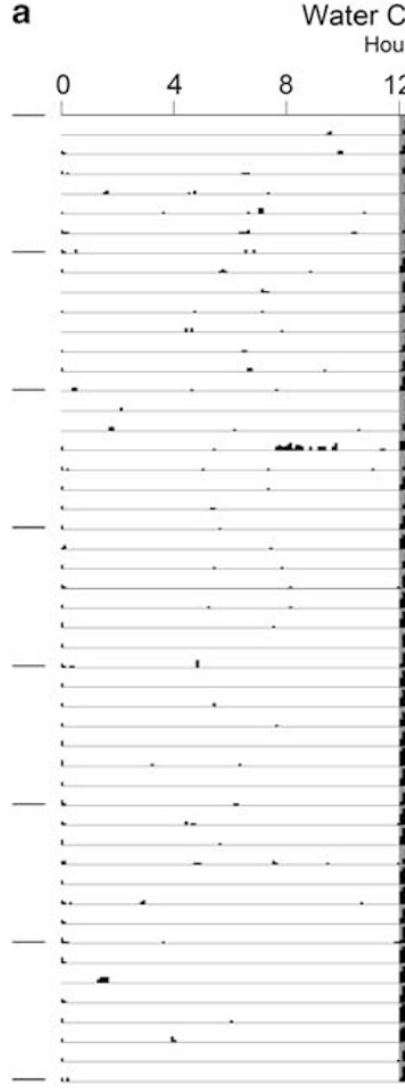

ter Control b

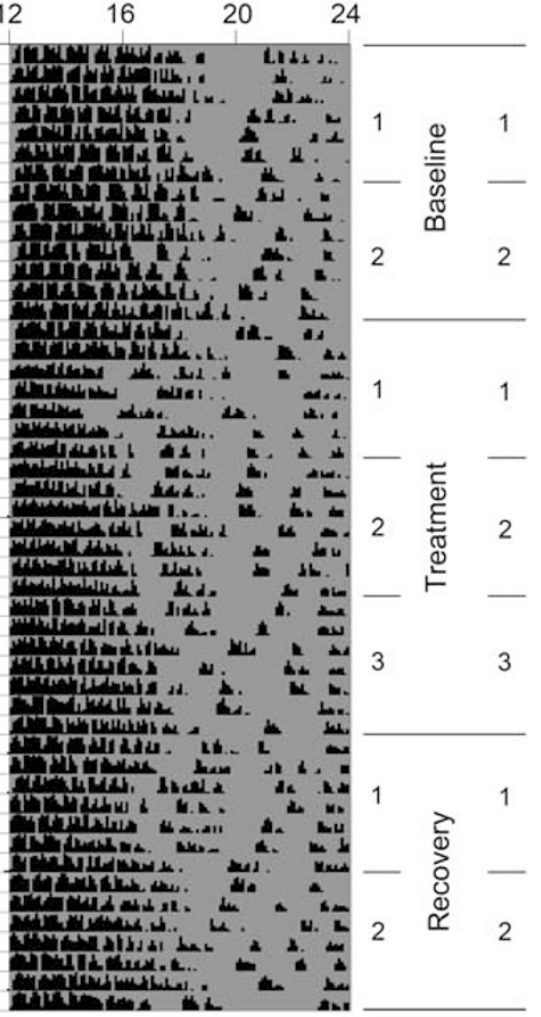

$\begin{array}{lllllll}0 & 4 & 8 & 12 & 16 & 20 & 24\end{array}$

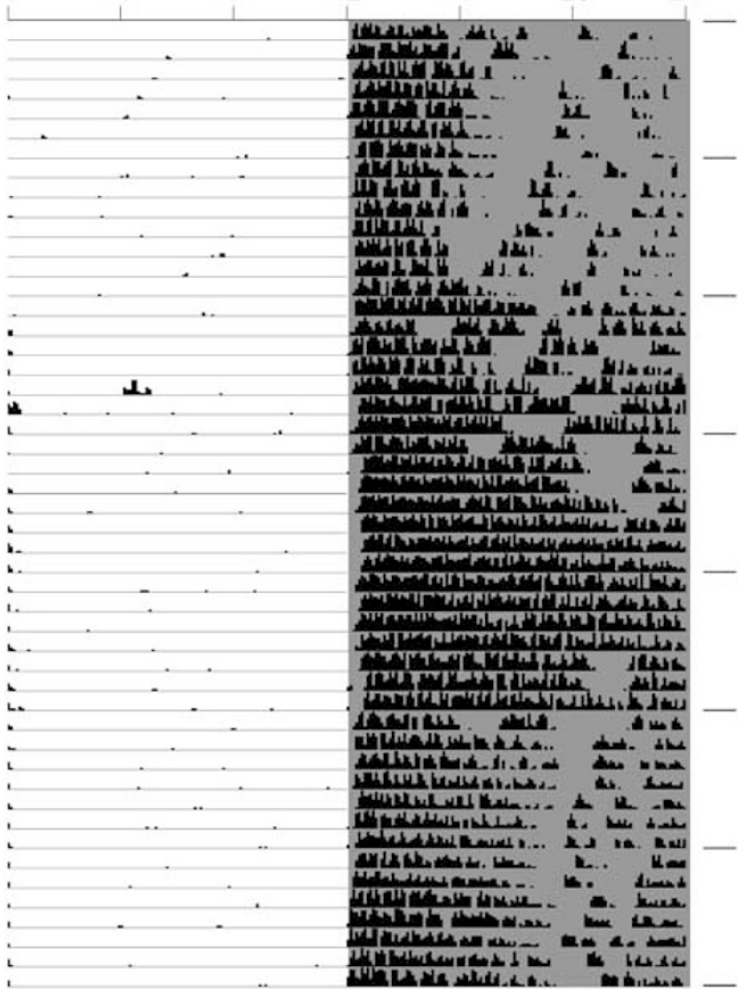

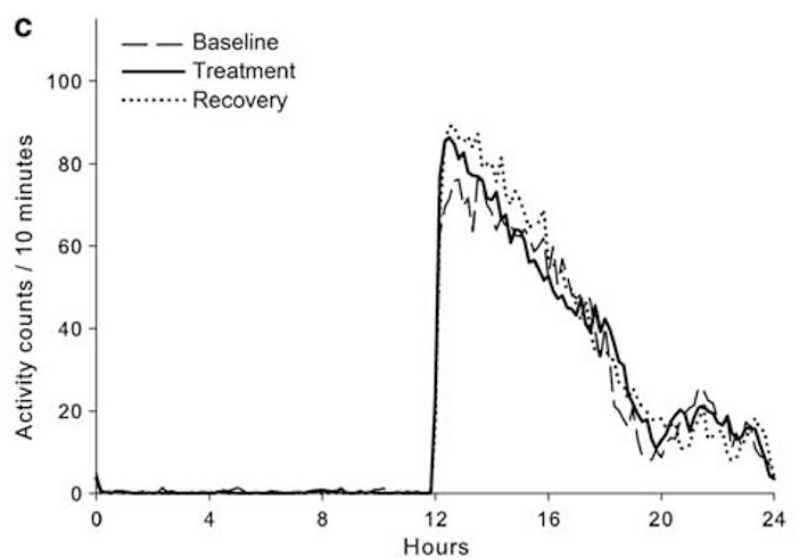

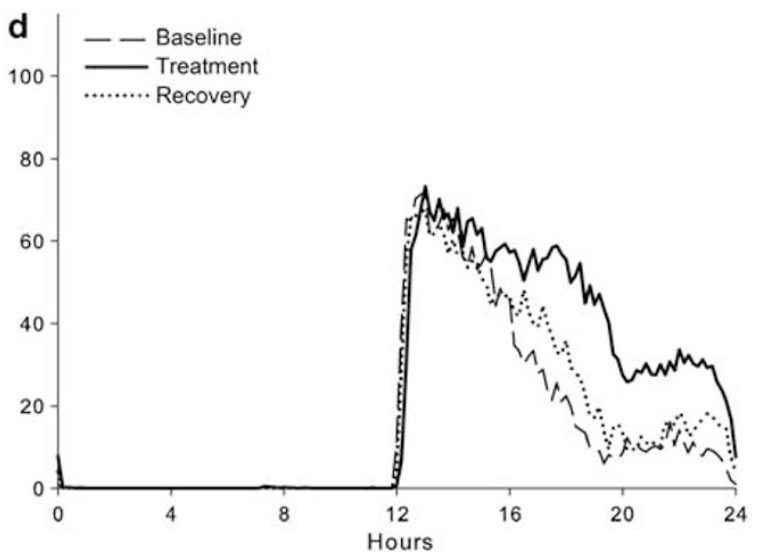

Figure I Methylphenidate increases locomotor activity in the mid-to-late night. Actograms depicting wheel-running behavior in animals given drinking water control (a) or methylphenidate in drinking water $(0.08 \%, b)$. Each horizontal line represents I day, with subsequent days plotted below the previous day. Black vertical lines denote times when the running wheel rotated, with the height of the line being proportional to the number of rotations. Gray shading indicates times of darkness, starting at hour 12. During baseline and recovery, animals were given normal drinking water. Average activity waveforms from water control (c) or methylphenidate $(0.08 \%, d)$ treated animals depicting the activity profile from each of the three portions of the study (Baseline, dashed line; Treatment, solid line; Recovery, dotted line). Quantification of activity for water control (c) or methylphenidate (0.08\%, d) treated animals from each week during the experiment revealed significant increases in activity in the mid and late night in only the methylphenidate-treated animals.

condition. While control animals exhibited a shortening of their free-running period over the first few weeks of the experiment, methylphenidate animals differed significantly from this pattern, exhibiting a lengthening of the freerunning period during methylphenidate treatment. This pattern was observed in both animals recorded using running wheels (treatment $\times$ time interaction, $F_{(1,9)}=$ $16.928, p=0.003$; Figure $3 \mathrm{a}-\mathrm{c}$ ) and with PIRs (treatment $\times$ time interaction, $F_{(1,10)}=14.801, p=0.003$; Figure $\left.3 \mathrm{~d}\right)$. An additional analysis revealed a dose-dependent effect, where 0.08 and $0.12 \%$ methylphenidate produced period lengthening significantly different from water control treatment (treatment $\times$ time interaction, $F_{(4,15)}=6.448, \quad p=0.003$; Figure $3 c$ ). In all, 0.02 and $0.04 \%$ methylphenidate produced trends toward longer periods than control animals, but these differences were non-significant, likely due to the small sample size $(n=3$ each). While the period shortened in animals with running wheels following the end of 


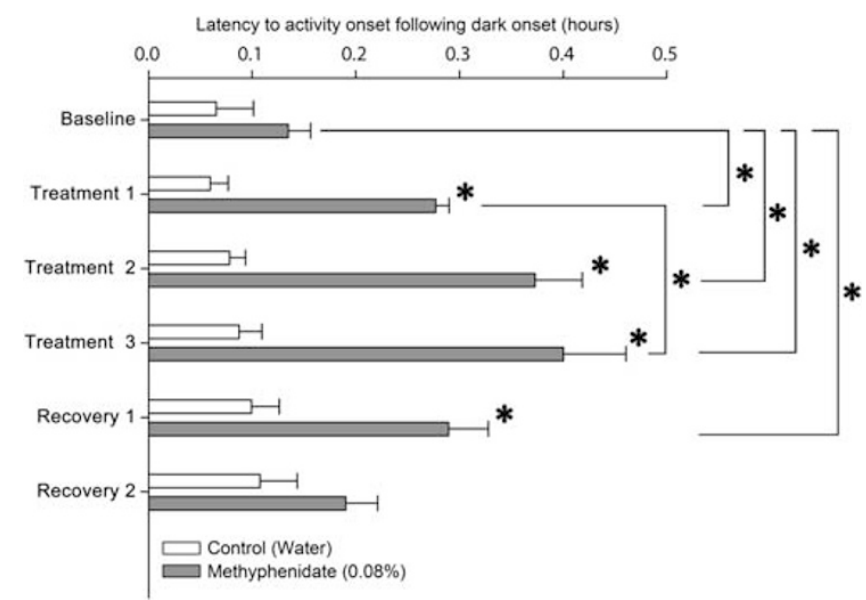

Figure 2 Methylphenidate significantly delays the onset of activity relative to the time of dark onset. The phase of activity onset in water control animals (white bars) was stable over the course of the experiment, while methylphenidate-treated animals (gray bars) exhibited progressively later activity onsets over successive weeks of treatment. $* p<0.05$.

methylphenidate treatment, no such change was observed in animals in cages equipped with PIRs.

Overall activity levels were significantly increased in animals in DD during treatment with methylphenidate. This was the case for both animals in cages equipped with running wheels (treatment $\times$ time interaction, $F_{(2,14)}=3.82$, $p<0.05$; Figure $3 \mathrm{e}$ ) and in cages equipped with PIRs (treatment $\times$ time interaction, $F_{(2,20)}=31.958, \quad p<0.001$; Figure 3f).

\section{Sleep}

Methylphenidate increased the amount of waking at the expense of NREM sleep, whereas REM sleep was not affected (Figure $4 \mathrm{a}$ and $\mathrm{b}$ ). The largest difference between treatment and control was found at the beginning of the light period, where animals on methylphenidate showed increased waking similar to dark period levels and a delay in the onset of the main sleep period (Figure 4a). SWA in NREM sleep showed the normal pattern of a gradual decrease in the light period and an increase in the course of the dark period under both conditions; however, under methylphenidate a delayed peak in SWA was observed in the second hour of the light period (Figure 4c). No differences between conditions were observed in the response to sleep deprivation or in the baseline EEG power density spectra $(0.25-25 \mathrm{~Hz})$ of the different vigilance states (data not shown).

\section{In-Vivo Electrophysiology}

SCN electrical activity rhythms were successfully recorded in nine wild-type animals (Figure 5a). Methylphenidate treatment significantly increased the amplitude of the SCN electrical activity rhythm (Figure 5b; $p<0.05$ ), with an average increase in the amplitude of $29 \%$. The trough of electrical SCN activity was significantly delayed from $5.32 \pm 0.28 \mathrm{~h}$ during baseline to $6.08 \pm 0.38 \mathrm{~h}$ during methylphenidate treatment (Figure $5 c$ ). An acute decrease in variability was observed during the first day of methylphenidate treatment $(p<0.05)$.

\section{DISCUSSION}

People with ADHD often exhibit sleep problems consistent with a phase delayed circadian clock (Boonstra et al, 2007; Owens et al, 2009; Sobanski et al, 2008; Van Veen et al, 2010). Some studies suggest that methylphenidate, a common pharmacotherapy for ADHD, may exacerbate these sleep problems (Corkum et al, 2008; Galland et al, 2010; Ironside et al, 2010; Sangal et al, 2006; Schwartz et al, 2004). We show here that in healthy adult mice, methylphenidate alone can alter both sleep and circadian rhythmicity. Activity levels are increased, particularly in the mid-to-late part of the active phase, thereby altering the circadian activity rhythm waveform. Methylphenidatetreated animals started their activity significantly later relative to dark onset than did control animals. Wake time was lengthened and sleep onset at the beginning of the light period was significantly delayed in methylphenidate-treated animals. When housed in constant darkness, the freerunning period of both wheel-running and general locomotor rhythms lengthened relative to their baseline period. Methylphenidate treatment also altered SCN electrical activity, producing a delayed trough, increased amplitude, and reduced variability. Changes in the SCN MUA rhythm, specifically the downward slope and trough, have been previously shown to correlate with behavioral activity (Houben et al, 2009), a pattern observed in the present study. All parameters of the SCN waveform taken together (trough, peak, rising, and downward slope) were also shifted, suggesting a delay in the SCN electrical activity rhythm. Furthermore, methylphenidate treatment produced an increase in the rhythm amplitude of SCN electrical activity. These findings show that methylphenidate can alter the circadian system, by affecting the properties of the central clock.

The changes in SCN activity could arise from the activation of neurotransmitter pathways, such as those that involve norepinephrine, or through methylphenidate altering activity levels that then alter clock properties. Activity can phase shift (Bobrzynska and Mrosovsky, 1998) and entrain (Edgar and Dement, 1991a; Marchant and Mistlberger, 1996) circadian rhythms. SCN firing rate is related to activity, with lower firing rate during bouts of spontaneous activity (Schaap and Meijer, 2001; van Oosterhout et al, 2012; Yamazaki et al, 1998). Increased activity has been shown to alter freerunning period (Deboer and Tobler, 2000; Edgar et al, 1991b; Mrosovsky, 1999; Yamada et al, 1988, 1990). Finally, scheduled confinement to a novel wheel typically leads to increased activity and can alter the phase angle of entrainment to a variety of lighting regimes (Sinclair and Mistlberger, 1997). Thus, the question arises by which route the SCN is affected. As behavioral activity is able to influence the clock, one possibility is that SCN activity is changed as a consequence of the behavioral changes. We can reject this option for the following reasons: first, while increased activity has been shown to alter free-running period, higher activity is associated with shorter periods (Deboer and Tobler, 2000; Edgar et al, 1991b; Mrosovsky, 1999; Yamada 
a

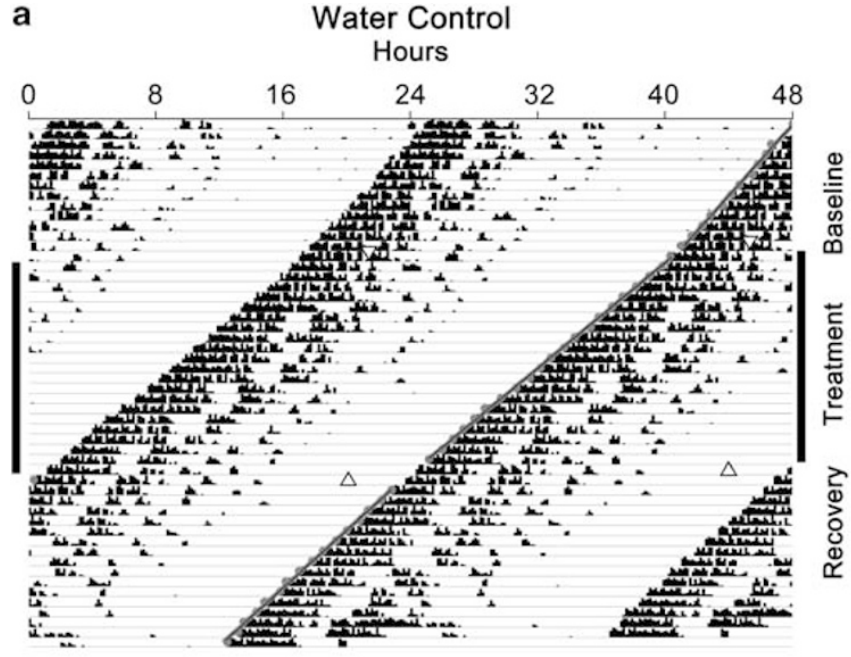

C
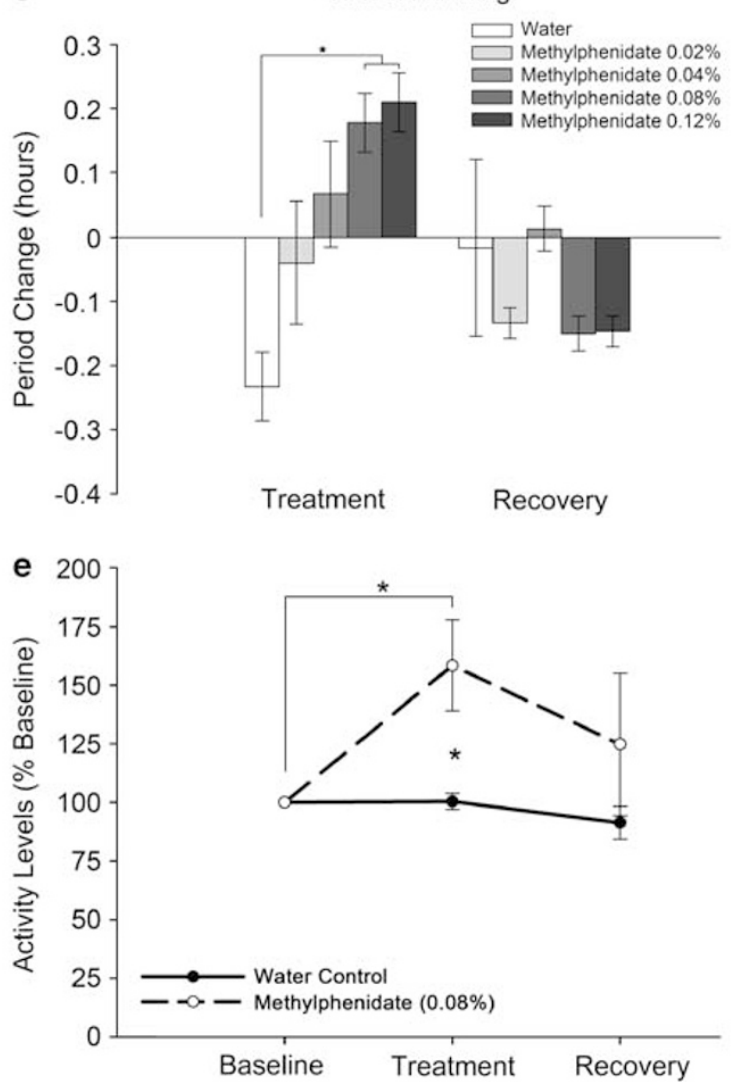

b

Methylphenidate $(0.08 \%)$ Hours

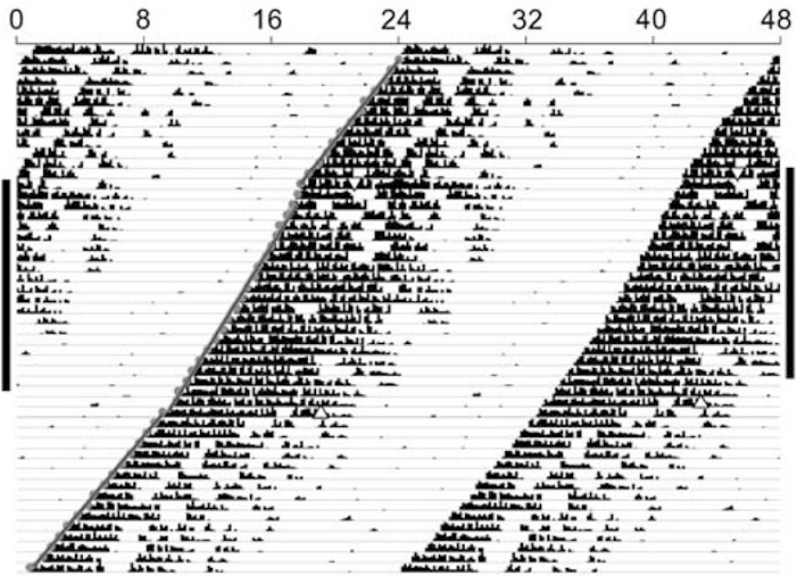

d

General Locomotion

Passive Infrared Detectors
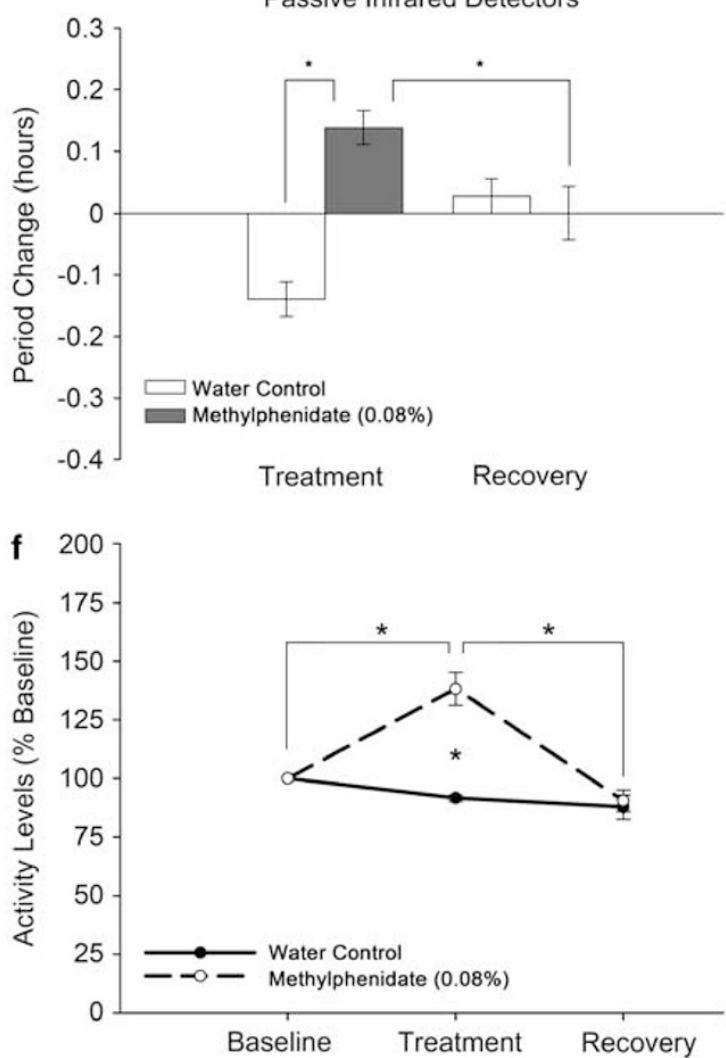

Figure 3 Representative double-plotted wheel-running actograms from water control (a) and methylphenidate (b) treated animals. The plotting convention is the same as for Figure I, except that each horizontal line represents 2 days of activity, with subsequent days plotted both to the right of and below the previous day. After 2 weeks of exposure to a light/dark cycle, the animals were transferred to constant darkness for 7 weeks, with the treatment phase (normal drinking water or $0.08 \%$ methylphenidate) beginning at the start of the third week (upside down white triangle) and ending at the end of the fifth week (upside right white triangle). Methylphenidate significantly lengthens the free-running period of locomotor circadian rhythms measured with both running wheels (c) and passive infrared motion sensors (d). Whereas animals treated with normal drinking water (white bars) typically exhibit a shorter freerunning period over time in constant darkness, methylphenidate-treated animals (gray bars) exhibited lengthening of their period during treatment in a dosedependent manner (c). Following the end of treatment, most methylphenidate-treated animals in cages equipped with running wheels exhibited a shortening in their free-running period, a phenomenon not observed in methylphenidate-treated animals in cages equipped with passive infrared motion sensors. Data contributing to the period change were calculated as period during the denoted bin (Treatment or Recovery) minus the period in the previous bin (Baseline or Treatment, respectively). $* 0<0.05$. Methylphenidate treatment significantly increases total wheel-running activity (e) and general locomotor activity as assessed with passive infrared motion sensors $(f)$ in animals housed in constant darkness. While activity levels of water-treated control animals (solid lines with filled circles) remained relatively stable over the experiment, methylphenidate-treated animals (dashed lines with open circles) were significantly elevated during treatment relative to baseline, and then dropped significantly following cessation of treatment. $* p<0.05$. 

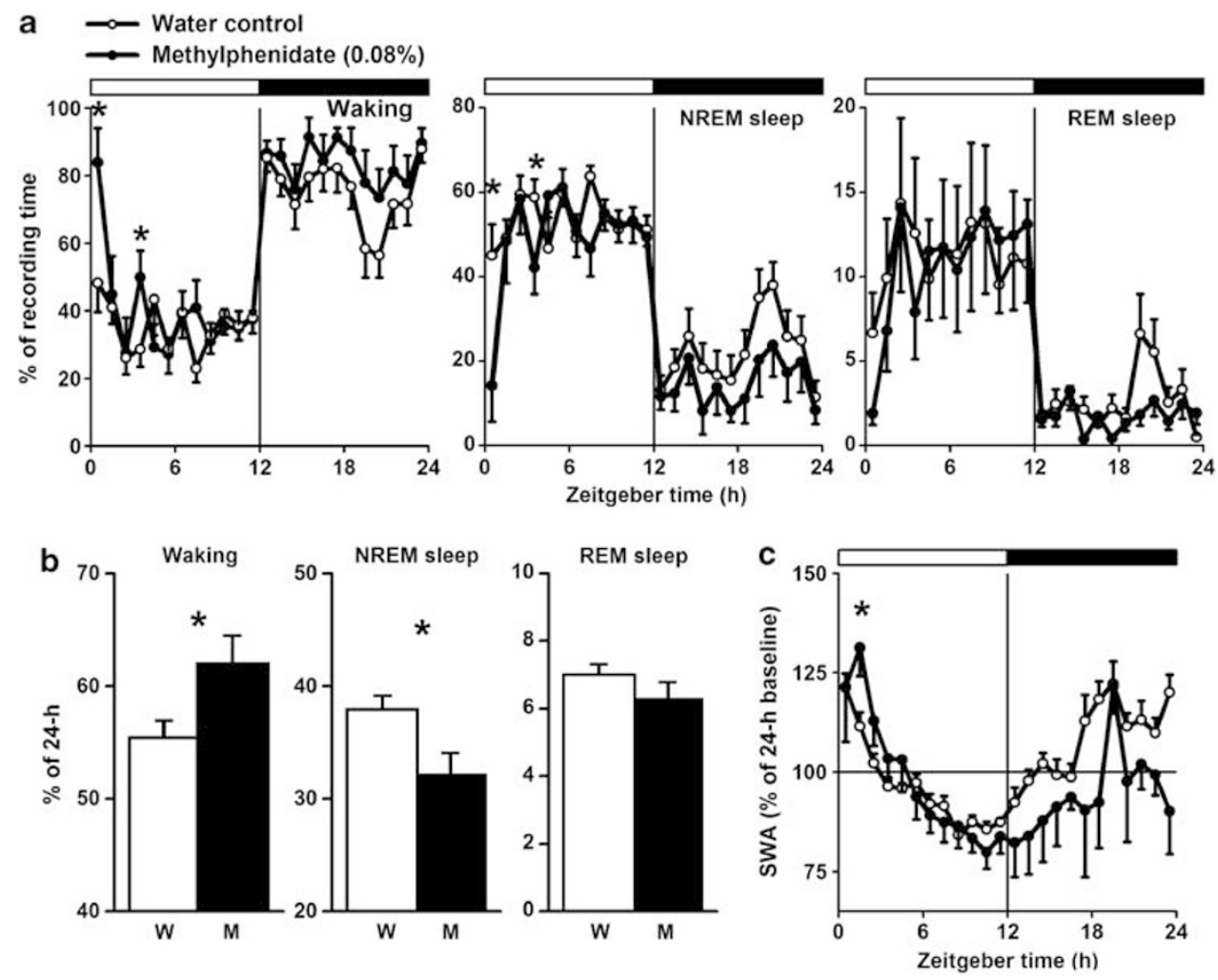

Figure 4 Methylphenidate (M) significantly changes the distribution (a) and total amount (b) of waking and non-rapid eye-movement (NREM) sleep compared with water control (W). Peak slow-wave activity in NREM sleep is delayed by I h, but the time course is similar to water control (c). ${ }^{*} p<0.05$.

et al, 1988, 1990). In our study the opposite was observed, with a lengthening of free-running period in the methylphenidate-treated animals. Furthermore, this lengthening of freerunning period was also observed in animals housed in cages without running wheels where high amplitude activity was more limited. Finally, activity levels returned to baseline immediately after the end of treatment, but free-running period either recovered incompletely in animals with running wheels, or remained stable at the lengthened period in animals without running wheels, suggesting that the change in period during treatment was actually due to enduring changes of the clock, rather than simply an acute response to increased activity levels.

The lengthening of the free-running period in constant darkness and the delay in sleep and activity rhythms is consistent with a delayed phase angle of entrainment. In addition, the trough in SCN electrical activity is also delayed. With a longer endogenous period, animals would need smaller daily delays of the circadian clock to remain entrained to the light-dark cycle. As such, a delayed phase angle of entrainment would expose less of the delay portion of the phase response curve to light at dusk. While the change in phase angle of entrainment is consistent with a change in free-running period, we cannot exclude the possibility that methylphenidate may alter phase angle of entrainment by altering circadian responses to light either by altering monoamine tone in the SCN or by altering activity levels. Both monoamines and activity have been shown to attenuate phase shifts to light (Antle et al, 2003; Mistlberger and Antle, 1998; Rea and Pickard, 2000). The observation that phase angle required over a week to return to baseline after the end of methylphenidate indicates that the phase of the circadian clock was delayed. The change in phase angle is also consistent with findings from placebocontrolled and baseline-comparison human studies examining the effects of methylphenidate on various sleep and circadian markers. Longer sleep latency and decreased total sleep time were observed in people given methylphenidate (Boonstra et al, 2007; Corkum et al, 2008; Galland et al, 2010; Sangal et al, 2006; Schwartz et al, 2004). A phase delay in circadian locomotor rhythms has also been noted (Ironside et al, 2010). These all point to an alteration in the underlying circadian clock, and are consistent with the delay of the phase angle of entrainment observed in the restactivity cycle and in sleep.

Changes to level and distribution of activity have been noted to single and daily injections of methylphenidate. Activity is altered in a dose-dependent manner, with lowto-moderate doses producing activity above baseline for $1-3 \mathrm{~h}$, although high doses $(40 \mathrm{mg} / \mathrm{kg})$ lead to a delayed onset of increased locomotor activity while animals first engage in stereotypic behavior (Gaytan et al, 1997). Daily injections for a week alter the amplitude, mean, and acrophase of activity rhythms (Lee et al, 2009, 2011), although the findings of these studies lacked the temporal resolution for detecting the behavioral phenomena described here. Similarly to other amphetamines, methylphenidate has been shown to restore circadian rhythmicity to animals rendered arrhythmic with SCN lesions (Honma and Honma, 1992), although only when activity levels were 

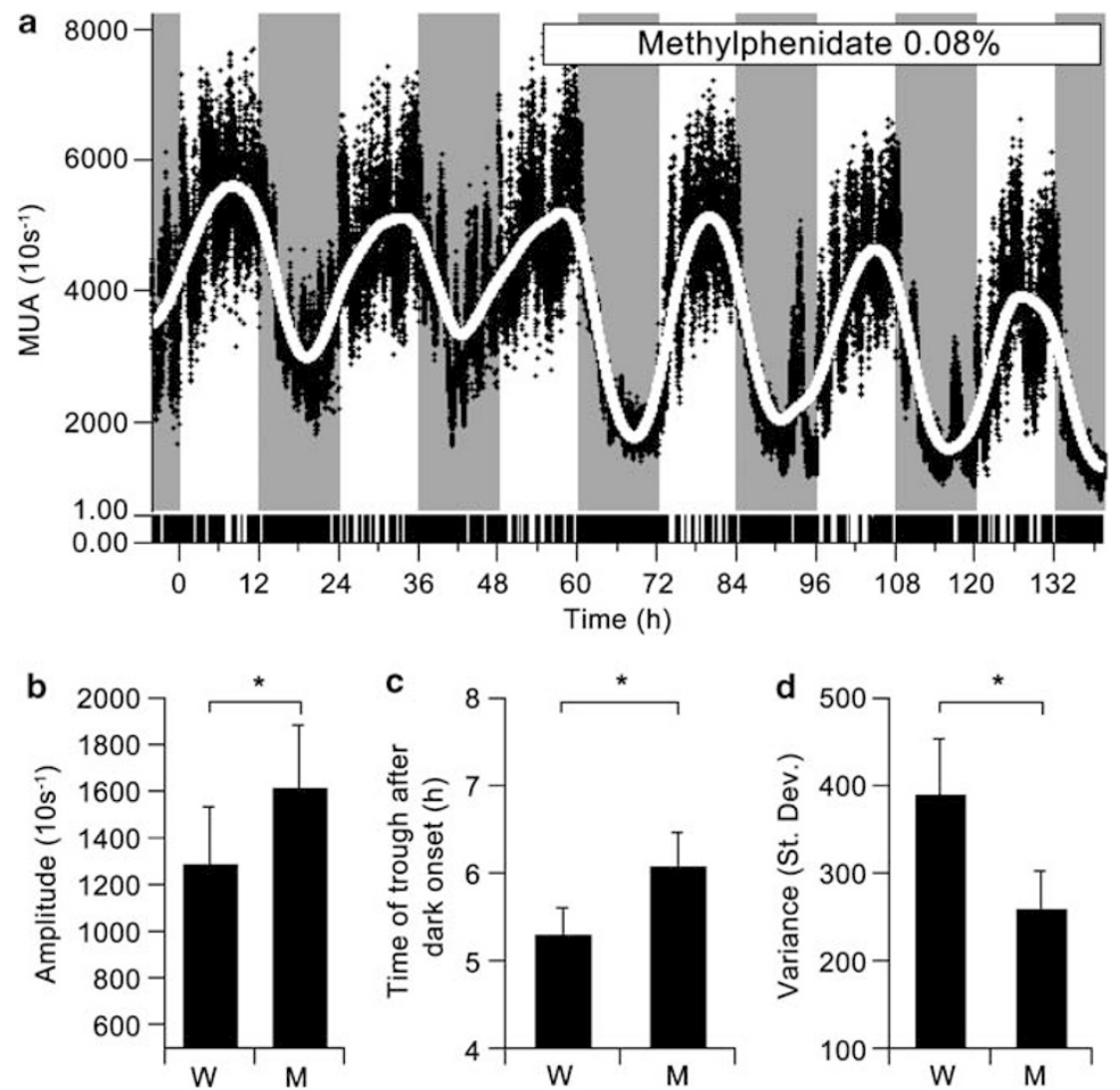

Figure 5 Representative SCN MUA recordings of a freely moving mouse in a 12:12 LD cycle (white and gray shading) during baseline and methylphenidate treatment (a). Individual data points represent 10 s epochs. Smoothed data are indicated by a white line. Behavioral activity was measured by passive infrared sensors and plotted at the bottom of the graph. Methylphenidate treatment (M) significantly increased the amplitude of the MUA rhythm compared with water control in baseline $(\mathrm{W})(\mathrm{b})$. The trough of the MUA rhythms is significantly delayed when switched from baseline to methylphenidate treatment (c). During the first day of treatment, the variance of the MUA signal is significantly decreased (d). ${ }^{*} p<0.05$.

double those observed during baseline. These effects in arrhythmic animals are thought to be due to the participation of a methamphetamine-sensitive circadian oscillator (MASCO) that is separate and distinct from the SCN. Our experiments differ essentially from experiments aimed to understand the MASCO (Honma et al, 1986; Honma and Honma, 1992). In these experiments, amphetamines were provided to SCN lesioned animals, while in our present study, the experiments were aimed to understand the influence of an amphetamine derivative on the SCN in healthy intact animals. When methamphetamine is given to SCN intact rats, it alters circadian rhythmicity to a much greater extent than was observed here, with large delays to the phase angle of entrainment or even failure to entrain (Honma et al, 1986). It is possible that the results observed in those studies, as well as in the current study, may be due to an interaction between the circadian clock in the SCN and the MASCO. Recently, atomoxetine, another drug used in the treatment of ADHD that shows greater selectivity for the noradrenergic system, was shown to phase shift the circadian clock when given as a single dose (O'Keeffe et al, 2012). The direction of phase shift observed depended on lighting condition, in that administration during the mid subjective day caused large delays in constant light and small advances in constant dark. Preliminary findings from our laboratory examining phase shifts to single doses of methylphenidate detected no phase shifts at any phase in constant dark (data not shown).

Methylphenidate delayed the onset of the main sleep bout at the beginning of the light period and reduced the amount of sleep over $24 \mathrm{~h}$, matching findings reported in humans (Galland et al, 2010). Both effects were mainly found in NREM sleep whereas REM sleep was not changed significantly. At the beginning of the light period, SWA in NREM sleep showed a delayed peak compared with control. The latter is in accordance with normal sleep homeostatic responses to the increased waking and delayed sleep onset, indicating that sleep homeostasis was not affected by methylphenidate. The data suggest that methylphenidate treatment renders the animals less susceptible to homeostatic sleep pressure as the animals are more awake over $24 \mathrm{~h}$ and are able to extend their active period by $1 \mathrm{~h}$ into the light period. Methylphenidate blocks both dopamine and norepinephrine transporters, increasing levels of these neurotransmitters in the synapse (Kodo et al, 2010), which can explain the increased waking as both monoamines are known to have wake and arousal promoting effects (Espana and Scammell, 2011). As a consequence, the animals initiate their main sleep period approximately an hour later with a higher sleep pressure than control.

The treatment with methylphenidate was relatively brief in the present study, lasting 3 weeks in our behavioral 
experiments, 2 weeks in our sleep experiments, and 5 days in our SCN recordings. Importantly, the phase angle of entrainment continued to delay throughout treatment. Therefore, we may not have observed the full effects of methylphenidate on the circadian system that may develop during long-term treatment. Given that people prescribed methylphenidate take it multiple times a day for years, it would be interesting to examine how long-term treatment would alter circadian rhythmicity. If phase angle continued to delay even further, then this would be consistent with even more severe circadian disruptions that could contribute to even more severe insomnia. No change in activity levels was noted throughout the duration of treatment, suggesting that this phenomenon does not habituate, at least over the short term.

Various options for both dose and route of administration are possible. We observed a significant dose-dependent effect on free-running period. The minimal dose to produce a significant change in period also produced significant changes to other circadian parameters, sleep architecture and SCN electrical activity. The route of administration used here suffers from the fact that the final dose consumed is dependent on drinking behavior of the individual animal. However, it is less invasive and disruptive than delivery by repeated systemic injections or intravenous infusion through a catheter. Osmotic minipumps, while less disruptive, have a limited capacity and would provide continuous dosing. Our approach of delivery in drinking water is unlimited and occurs only when the mice are awake, more closely matching human consumption patterns. The estimated maximal ingested dose with a $0.08 \%$ methylphenidate solution $(\sim 150 \mathrm{mg} / \mathrm{kg} /$ day $)$ is much higher than that typically used in humans, although the pharmacokinetics are dramatically different between mice and humans (Manjanatha et al, 2008). Similar trends to the observed effect on circadian period were observed at lower doses, but the magnitude of effect was smaller, and the low sample size reduced statistical power. In comparison with other mouse studies, it is important to note that our dose is the cumulative amount consumed over $24 \mathrm{~h}$, while many other studies in mice examining behavior use a single injection (eg, Zhou et al, 2010).

Given the alteration in free-running period, timing of sleep, and phase angle of entrainment, it will be important to determine how circadian responses to light are altered. Acute doses of atomoxetine alter responses to phase shifting by light, as well as light-induced expression of a number of clock genes and immediate early genes in the SCN (O'Keeffe et al, 2012). Acute atomoxetine alters clock gene and immediate early gene expression in the SCN at phases when atomoxetine alone produces phase shifts, suggesting that it not only alters light input, but also the underlying transcription-translation feedback loops that give rise to the intracellular circadian clock. Given the changes observed here, it is likely that methylphenidate will also alter the molecular clock, possibly changing the acrophase, amplitude, or spatiotemporal patterns of gene expression in the SCN.

Patients with ADHD often exhibit sleep problems, with sleep-onset insomnia being a common complaint. While methylphenidate manages many ADHD symptoms, it appears to exacerbate the current sleep problems these patients experience. Given the changes observed here, it is likely that chronic methylphenidate treatment alters the circadian clock in such a way so as to promote sleep-onset insomnia. Many of the symptoms of ADHD are similar to what people experience following poor sleep. It has been suggested (Corkum et al, 2008) that the sleep problems may contribute to the severity of the ADHD symptoms. If this is true, then it is possible that methylphenidate may also contribute to ADHD symptoms by decreasing the quality of sleep. A thorough screen of other ADHD medications may reveal better alternatives for managing ADHD symptoms that do not alter the underlying clock in such a way so as to increase the likelihood and severity of insomnia. Conversely, the present results on phase angle of entrainment, free-running period, and delayed sleep onset suggest that methylphenidate may be a useful treatment for circadian sleep disorders such as advanced phase sleep syndrome.

\section{ACKNOWLEDGEMENTS}

MCA's research is supported by a Discovery grant from the Natural Sciences and Engineering Research Council of Canada. JHM's research is supported by Technology Foundation STW, P10-18. TD's research was supported by the Netherlands Organization for Scientific Research (NWO, grant 818.02.016).

\section{DISCLOSURE}

The authors declare that, except for income received from their primary employer, no financial support or compensation has been received from any individual or corporate entity for research or professional service and there are no personal financial holdings that could be perceived as constituting a potential conflict of interest.

\section{REFERENCES}

Achermann P, Borbely AA (2011). Sleep homeostasis and models of sleep regulation. In: Kryger MH, Roth T, Dement WC (eds). Principles and Practice of Sleep Medicine 5th edn. Elsevier Saunders: St Louis, MO, pp 431-444.

Algahim MF, Yang PB, Wilcox VT, Burau KD, Swann AC, Dafny N (2009). Prolonged methylphenidate treatment alters the behavioral diurnal activity pattern of adult male Sprague-Dawley rats. Pharmacol Biochem Behav 92: 93-99.

Antle MC, Ogilvie MD, Pickard GE, Mistlberger RE (2003). Response of the mouse circadian system to serotonin $1 \mathrm{~A} / 2 / 7$ agonists in vivo: surprisingly little. J Biol Rhythms 18: 145-158.

Antle MC, Silver R (2005). Orchestrating time: arrangements of the brain circadian clock. Trends Neurosci 28: 145-151.

Bobrzynska KJ, Mrosovsky N (1998). Phase shifting by noveltyinduced running: activity dose-response curves at different circadian times. J Comp Physiol A 182: 251-258.

Boonstra AM, Kooij JJ, Oosterlaan J, Sergeant JA, Buitelaar JK, Van Someren EJ (2007). Hyperactive night and day? Actigraphy studies in adult ADHD: a baseline comparison and the effect of methylphenidate. Sleep 30: 433-442.

Corkum P, Panton R, Ironside S, Macpherson M, Williams T (2008). Acute impact of immediate release methylphenidate administered three times a day on sleep in children with attention-deficit/hyperactivity disorder. J Pediatr Psychol 33: 368-379. 
Deboer T, Detari L, Meijer JH (2007a). Long term effects of sleep deprivation on the mammalian circadian pacemaker. Sleep 30: 257-262.

Deboer T, Ruijgrok G, Meijer JH (2007b). Short light-dark cycles affect sleep in mice. Eur J Neurosci 26: 3518-3523.

Deboer T, Tobler I (2000). Running wheel size influences circadian rhythm period and its phase shift in mice. J Comp Physiol A 186: 969-973.

Edgar DM, Dement WC (1991a). Regularly scheduled voluntary exercise synchronizes the mouse circadian clock. Am J Physiol 261(4 Pt 2): R928-R933.

Edgar DM, Martin CE, Dement WC (1991b). Activity feedback to the mammalian circadian pacemaker: influence on observed measures of rhythm period length. J Biol Rhythms 6: 185-199.

Eilers PH (2003). A perfect smoother. Anal Chem 75: 3631-3636.

Espana RA, Scammell TE (2011). Sleep neurobiology from a clinical perspective. Sleep 34: 845-858.

Galland BC, Tripp EG, Taylor BJ (2010). The sleep of children with attention deficit hyperactivity disorder on and off methylphenidate: a matched case-control study. J Sleep Res 19: 366-373.

Gaytan O, Ghelani D, Martin S, Swann A, Dafny N (1997). Methylphenidate: diurnal effects on locomotor and stereotypic behavior in the rat. Brain Res 777: 1-12.

Honma K, Honma S, Hiroshige T (1986). Disorganization of the rat activity rhythm by chronic treatment with methamphetamine. Physiol Behav 38: 687-695.

Honma S, Honma K (1992). Locomotor rhythms induced by methylphenidate in suprachiasmatic nuclei-lesioned rats. Neurosci Lett 137: 24-28.

Houben T, Deboer T, van Oosterhout F, Meijer JH (2009). Correlation with behavioral activity and rest implies circadian regulation by SCN neuronal activity levels. J Biol Rhythms 24: 477-487.

Huber R, Deboer T, Tobler I (2000). Effects of sleep deprivation on sleep and sleep EEG in three mouse strains: empirical data and simulations. Brain Res 857: 8-19.

Ironside S, Davidson F, Corkum P (2010). Circadian motor activity affected by stimulant medication in children with attentiondeficit/hyperactivity disorder. J Sleep Res 19: 546-551.

Kissling C, Retz W, Wiemann S, Coogan AN, Clement RM, Hunnerkopf $\mathrm{R}$ et al (2008). A polymorphism at the 3'untranslated region of the CLOCK gene is associated with adult attention-deficit hyperactivity disorder. Am J Med Genet B Neuropsychiatr Genet 147: 333-338.

Kodo K, Ago Y, Cong Y, Kita Y, Takuma K, Matsuda T (2010). Effects of acute and chronic administration of atomoxetine and methylphenidate on extracellular levels of noradrenaline, dopamine and serotonin in the prefrontal cortex and striatum of mice. J Neurochem 114: 259-270.

Lee MJ, Yang PB, Wilcox VT, Burau KD, Swann AC, Dafny N (2009). Does repetitive Ritalin injection produce long-term effects on SD female adolescent rats? Neuropharmacology 57: 201-207.

Lee MJ, Yang PB, Wilcox VT, Burau KD, Swann AC, Dafny N (2011). Repetitive methylphenidate administration modulates the diurnal behavioral activity pattern of adult female SD rats. J Neural Transm 118: 285-298.

Manjanatha MG, Shelton SD, Dobrovolsky VN, Shaddock JG, McGarrity LG, Doerge DR et al (2008). Pharmacokinetics, doserange, and mutagenicity studies of methylphenidate hydrochloride in B6C3F1 mice. Environ Mol Mutagen 49: 585-593.

Marchant EG, Mistlberger RE (1996). Entrainment and phase shifting of circadian rhythms in mice by forced treadmill running. Physiol Behav 60: 657-663.
Mistlberger RE, Antle MC (1998). Behavioral inhibition of light-induced circadian phase resetting is phase and serotonin dependent. Brain Res 786: 31-38.

Mrosovsky N (1999). Further experiments on the relationship between the period of circadian rhythms and locomotor activity levels in hamsters. Physiol Behav 66: 797-801.

Novakova M, Paclt I, Ptacek R, Kuzelova H, Hajek I, Sumova A (2011). Salivary melatonin rhythm as a marker of the circadian system in healthy children and those with attention-deficit/ hyperactivity disorder. Chronobiol Int 28: 630-637.

O'Keeffe SM, Thome J, Coogan AN (2012). The noradrenaline reuptake inhibitor atomoxetine phase-shifts the circadian clock in mice. Neuroscience 201: 219-230.

Owens J, Sangal RB, Sutton VK, Bakken R, Allen AJ, Kelsey D (2009). Subjective and objective measures of sleep in children with attention-deficit/hyperactivity disorder. Sleep Med 10: 446-456.

Pilcher JJ, Huffcutt AI (1996). Effects of sleep deprivation on performance: a meta-analysis. Sleep 19: 318-326.

Rea M, Pickard G (2000). Serotonergic modulation of photic entrainment in the Syrian hamster. Biol Rhythm Res 31: 284-314.

Sadeh A (2007). Consequences of sleep loss or sleep disruption in children. Sleep Med Clin 2: 513-520.

Sangal RB, Owens J, Allen AJ, Sutton V, Schuh K, Kelsey D (2006). Effects of atomoxetine and methylphenidate on sleep in children with ADHD. Sleep 29: 1573-1585.

Schaap J, Meijer JH (2001). Opposing effects of behavioural activity and light on neurons of the suprachiasmatic nucleus. Eur $J$ Neurosci 13: 1955-1962.

Schwartz G, Amor LB, Grizenko N, Lageix P, Baron C, Boivin DB et al (2004). Actigraphic monitoring during sleep of children with ADHD on methylphenidate and placebo. J Am Acad Child Adolesc Psychiatry 43: 1276-1282.

Sinclair SV, Mistlberger RE (1997). Scheduled activity reorganizes circadian phase of Syrian hamsters under full and skeleton photoperiods. Behav Brain Res 87: 127-137.

Sobanski E, Schredl M, Kettler N, Alm B (2008). Sleep in adults with attention deficit hyperactivity disorder (ADHD) before and during treatment with methylphenidate: a controlled polysomnographic study. Sleep 31: 375-381.

Van der Heijden K, Smits M, Gunning W (2005). Sleep-related disorders in ADHD: a review. Clin Pediatr 44: 201-210.

van Oosterhout F, Lucassen EA, Houben T, vanderLeest HT, Antle MC, Meijer JH (2012). Amplitude of the SCN clock enhanced by the behavioral activity rhythm. PLoS One 7: e39693.

Van Veen MM, Kooij JJ, Boonstra AM, Gordijn MC, Van Someren EJ (2010). Delayed circadian rhythm in adults with attentiondeficit/hyperactivity disorder and chronic sleep-onset insomnia. Biol Psychiatry 67: 1091-1096.

Yamada N, Shimoda K, Ohi K, Takahashi S, Takahashi K (1988). Free-access to a running wheel shortens the period of freerunning rhythm in blinded rats. Physiol Behav 42: 87-91.

Yamada N, Shimoda K, Takahashi K, Takahashi S (1990). Relationship between free-running period and motor activity in blinded rats. Brain Res Bull 25: 115-119.

Yamazaki S, Kerbeshian MC, Hocker CG, Block GD, Menaker M (1998). Rhythmic properties of the hamster suprachiasmatic nucleus in vivo. J Neurosci 18: 10709-10723.

Zhou M, Rebholz H, Brocia C, Warner-Schmidt JL, Fienberg AA, Nairn AC et al (2010). Forebrain overexpression of CK1delta leads to down-regulation of dopamine receptors and altered locomotor activity reminiscent of ADHD. Proc Natl Acad Sci USA 107: 4401-4406. 